\title{
Tumor-Specific Binding of Radiolabeled PEGylated GIRLRG Peptide: A Novel Agent for Targeting Cancers
}

\author{
Vaishali Kapoor ${ }^{1}$, David Y.A. Dadey ${ }^{1,2}$, Kim Nguyen ${ }^{1,3}$, Scott A. Wildman ${ }^{4}$, Kelly Hoye ${ }^{5}$, Arpine Khudanyan ${ }^{1}$, \\ Nilantha Bandara ${ }^{1,3}$, Buck E. Rogers ${ }^{1,3}$, Dinesh Thotala ${ }^{1,6}$, and Dennis E. Hallahan ${ }^{1,3,6}$ \\ ${ }^{I}$ Department of Radiation Oncology, Washington University in St. Louis, St. Louis, Missouri; ${ }^{2}$ Medical Scientist Training Program, \\ Washington University in St. Louis, St. Louis, Missouri; ${ }^{3}$ Mallinckrodt Institute of Radiology, Washington University in St. Louis, \\ St. Louis, Missouri; ${ }^{4}$ Carbone Cancer Center, University of Wisconsin, Madison, Wisconsin; ${ }^{5}$ Medical Guidance Systems, St. Louis, \\ Missouri; and ${ }^{6}$ Siteman Cancer Center, School of Medicine, Washington University in St. Louis, St. Louis, Missouri
}

Cancer-specific targeting sparing normal tissues would significantly enhance cancer therapy outcomes and reduce cancer-related mortality. One approach is to target receptors or molecules that are specifically expressed on cancer cells. Peptides as cancer-specific targeting agents offer advantages such as ease of synthesis, low antigenicity, and enhanced diffusion into tissues. Glucose-regulated protein 78 (GRP78) is an endoplasmic reticulum stress chaperone that regulates the unfolded protein response and is overexpressed in various cancers. In this study, we evaluated GIRLRG peptide that specifically targets GRP78 for cancer-specific binding (in vitro) and noninvasive tumor imaging (in vivo). Methods: GIRLRG peptide was modeled into the GRP78 ATPase domain using computational modeling. Surface plasmon resonance studies were performed to determine the affinity of GIRLRG peptide to GRP78 protein. GIRLRG was conjugated with $P E G$ to prolong its circulation in mice. Tumor binding efficacy of PEG-GIRLRG peptide was evaluated in nude mice bearing heterotopic cervical (HT3), esophageal (OE33), pancreatic (BXPC3), lung (A549), and glioma (D54) tumors. Nano-SPECT/CT imaging of the mice was performed 48 and $72 \mathrm{~h}$ after injection with ${ }^{111} \mathrm{In}$-labeled PEG-GIRLRG or PEG-control peptide. Post-SPECT biodistribution studies were performed $96 \mathrm{~h}$ after injection of the radiolabeled peptides. Results: Using molecular modeling and surface plasmon resonance, we identified that GIRLRG was binding with an affinity constant of $2.16 \times 10^{-3} \mathrm{M}$ in the ATPase domain of GRP78. GIRLRG peptide specifically bound to cervical, lung, esophageal, and glioma cells. SPECT imaging revealed that ${ }^{111}$ In-PEG-GIRLRG specifically bound to cervical, esophageal, pancreatic, lung, and brain tumors. Post-SPECT biodistribution data also validated the SPECT imaging results. Conclusion: GIRLRG peptide specifically binds to the ATPase domain of GRP78. Radiolabeled PEG-GIRLRG could be used to target various cancers. Further studies would be required to translate PEG-GIRLRG peptide into the clinic.

Key Words: SPECT; GIRLRG peptide; GRP78; molecular imaging

J Nucl Med 2016; 57:1991-1997

DOI: 10.2967/jnumed.115.165118

Received Aug. 5, 2015; revision accepted Jun. 21, 2016.

For correspondence or reprints contact: Dennis E. Hallahan, Department of Radiation Oncology, Washington University School of Medicine in St. Louis, 4511 Forest Park, St. Louis, MO 63108

E-mail: dhallahan@radonc.wustl.edu

Dinesh Thotala, Department of Radiation Oncology, Washington University

School of Medicine in St. Louis, 4511 Forest Park, St. Louis, MO 63108.

E-mail: dthotala@radonc.wustl.edu

Published online Jul. 21, 2016.

COPYRIGHT (c) 2016 by the Society of Nuclear Medicine and Molecular Imaging, Inc.
O worldwide. Cancer treatments require precise spatial identification of tumors. Molecular imaging using SPECT or PET are efficacious imaging modalities for the identification of cancer-specific agents (1). Several radiolabeled targeting agents, including small molecules, peptides, proteins, and antibodies and antibody fragments, have been used in noninvasive imaging and specific detection of tumors. Molecular imaging using radiolabeled peptides offer several advantages over traditional imaging modalities. Targeting peptides are relatively small in size, allowing enhanced diffusion properties, target accessibility, and high sensitivity as imaging tracers. Additionally, peptides have little antigenicity and are economic to synthesize (2). Despite significant advances, there are few radiolabeled peptide-based tracers that have been approved for clinical use (2). There are several factors impeding the development of newer agents, including imaging efficacy and sensitivity, specificity, and translational ability. There is a need for developing novel cancer-specific targets and their peptide ligands, which could be developed as tracers for the noninvasive imaging of cancers.

We developed and characterized a novel peptide, GIRLRG, that binds specifically to tumors ex vivo and in vivo. The GIRLRG peptide was identified by in vivo phage-display biopanning and was shown to selectively recognize tumors that have been irradiated (3). We have previously demonstrated that GIRLRG conjugated to paclitaxel-encapsulated nanoparticles specifically targeted breast cancer (MDA-MB-231) and glioblastoma (GL261) (3). We further identified that GIRLRG specifically binds to glucose-regulated protein $78 \mathrm{kDa}$ (GRP78) (3). GRP78 is the major endoplasmic reticulum (ER) chaperone and is a biomarker for ER stress. GRP78 expression is elevated in various cancers, including lung, bladder, stomach, breast, and gastric, as well as gliomas due to glucose deprivation, hypoxia, and acidosis in the microenvironment of poorly vascularized tumors (4). In multiple tumor types, GRP78 overexpression leads to resistance to chemotherapy and knockdown of GRP78 leads to tumor cell sensitization to these treatments $(4,5)$. ER stress leads to induction of GRP78 in the ER compartment, as well as relocalization from the ER to the cell surface (6). Cell surface GRP78 is detected on many types of cancer cells in vivo $(7,8)$ and is not usually present on normal cells (4,9-11). Expression of GRP78 on the surface of human cancer cells is associated with tumorigenesis, tumor progression, angiogenesis, and metastasis, thereby demonstrating its utility as an anticancer therapeutic target (4). GRP78 is not confined to the plasma membrane or ER compartments. GRP78 can be shed and has 
been reported in the serum of non-small cell lung cancer patients and culture supernatants of prostate cancer (PC-3) and colon cancer (HRT-18) cells $(12,13)$. Recently, cell surface GRP78 was reported to be a promising target for antibodies and peptides $(9,11,14,15)$.

In this study, we identified the binding site and affinity of GIRLRG peptide to GRP78. Using heterotopic tumor models in mice, we found that GIRLRG peptide specifically binds to tumors and it could be potentially developed as an imaging agent for various cancers.

\section{MATERIALS AND METHODS}

\section{Cell Lines}

A549 cells are adenocarcinomas derived from human alveolar basal epithelial cells (16). D54 cells were derived from a grade IV glioblastoma multiforme (17). Both A549 and D54 cells were cultured in Dulbecco modified Eagle medium/F12 medium. The cell line OE33, also known as JROECL33, was established from the adenocarcinoma of the lower esophagus (18). BxPC3 cells were derived from a primary adenocarcinoma of the pancreas (19). Both OE33 and BxPC3 cells were cultured in RPMI 1640 medium. HT3 cells were derived from a metastatic site of cervical cancer (20). These cells were cultured in Iscove's modified Dulbecco media. All media were supplemented with $10 \%$ fetal bovine serum, penicillin, and streptomycin. The cells were maintained at $37^{\circ} \mathrm{C}$ in a $5 \% \mathrm{CO}_{2}$ incubator.

\section{Peptide Synthesis}

DTPA-PEG-(KKK)-GIRLRG (mPEG40K-carbonyl-Lys(CHX-A" DTPA)-AEEAc-Lys(CHX-A" -DTPA)-AEEAc-Trp-Lys(CHX-A" DTPA)-AEEAc-Gly-Ile-Arg-Leu-Arg-Gly-OH trifluoroacetate salt) and DTPA-PEG-(KKK)-control (mPEG40K-carbonyl-Lys(CHX-A" -DTPA)AEEAc-Lys(CHX-A" -DTPA)-AEEAc-Trp-Lys(CHX-A" -DTPA)-OH) containing $40 \mathrm{kDa}$ PEG were synthesized by Bachem (USA). Fluorescein isothiocyanate (FITC)-GIRLRG was synthesized by China Peptides using standard solid-phase Fmoc chemistry. The peptides were purified to a minimum purity of $95 \%$ by high-performance liquid chromatography and were isolated by lyophilization.

\section{Molecular Modeling Studies}

The GIRLRG peptide was modeled into the GRP78 ATPase domain, starting from the published crystal structure with adenosine diphosphate bound, 3IUC (21). Adenosine diphosphate and associated solvent molecules were removed, and the apo structure was minimized using the Amber99 force field (22) in the Molecular Operating Environment software (version 2011.10; Chemical Computing Group Inc.). The GIRLRG peptide was placed manually into the groove between Leu 84 and Arg 289 (residue numbering from 3IUC), with the first Arg placed into the adenosine binding site. The orientation was selected on the basis of the observation that when modifying the sequence of GIRLRG, specifically the first Arg was consistently required for binding. The resulting binding mode was again minimized with the Amber99 force field. Additional minimization by molecular dynamics methods (for use in subsequent efforts) showed no significant change in complex structure.

\section{Surface Plasmon Resonance (SPR)}

Affinity of GIRLRG for GRP78 protein was measured by the biosensor-based SPR technique using an automatic apparatus BIAcore 2000 (GE Healthcare) as described earlier (23). The recombinant eukaryotic GRP78 protein (Prospec, USA) was immobilized by amine coupling on the CM4 sensor surface (ligand), and GIRLRG peptide was used as the analyte. Experiments were performed at $25^{\circ} \mathrm{C}$ in 4-(2-hydroxyethyl)-1-piperazineethanesulfonic acid (HEPES) buffer saline-ethylenediaminetetraacetic acid (EDTA) surfactant P20 (HBSEP) buffer (GE Healthcare). GRP78 protein was immobolized using surface preparation wizard for amine coupling. Briefly, equal volumes $(115 \mu \mathrm{L})$ of $N$-hydroxysuccinimide $(2.3 \mathrm{mg}$ in $200 \mu \mathrm{L}$ of water) and $N$-ethyl- $N^{\prime}-3$ (diethylamino propyl) carbodiimide ( $15 \mathrm{mg}$ in $200 \mu \mathrm{L}$ of water) were mixed, and $75 \mu \mathrm{L}$ of this solution were injected into the flow cell at the flow rate of $5 \mu \mathrm{L} / \mathrm{min}$ across the CM4 sensor chips to activate the carboxy methylated dextran surface for $15 \mathrm{~min}$. GRP78 protein $(50 \mu \mathrm{g} / \mathrm{mL}$ in $10 \mathrm{mM}$ sodium acetate, $\mathrm{pH} 4.7)$ was injected at the flow rate of $5 \mu \mathrm{L} / \mathrm{min}$ across the activated surface for $25 \mathrm{~min}$. The residual $N$-hydroxysuccinimide esters were inactivated with ethanolamine $(50 \mu \mathrm{L})$ for $10 \mathrm{~min}$. A blank reference surface was also prepared with the same procedure by activation with $N$-ethyl- $N^{\prime}-3$ (diethylamino propyl) carbodiimide and $N$-hydroxysuccinimide and then inactivation with ethanolamine. The affinity of the interaction was determined from the level of binding at equilibrium as a function of the sample concentrations by BIA evaluation software 3.0. The rate constant was obtained by fitting the sensogram data after reference subtraction (data from the blank channel) using the BIA evaluation 3.0 software.

\section{Immunofluorescence}

Cancer cells (A549, HT3, D54, and OE33) were grown on chamber slides (Millipore, USA) and incubated with FITC-GIRLRG peptide $\left(10 \mu \mathrm{g} / \mathrm{mL}\right.$ ) for $2 \mathrm{~h}$ at $37^{\circ} \mathrm{C}$ in a $\mathrm{CO}_{2}$ incubator. The cells were then washed with phosphate-buffered saline to remove unbound peptide and fixed with $4 \%$ paraformaldehyde at room temperature. The nuclei were stained with 4',6-diamidino-2-phenylindole, and fluorescent images were captured using a Carl Zeiss microscope.

\section{Radiolabeling of Peptides}

The radiolabeling procedure was optimized by varying the $\mathrm{pH}$, buffers, temperature, and amounts of ${ }^{111} \mathrm{InCl}_{3}$ added per mg of DTPA-PEG compounds. DTPA-PEG control and DTPA-PEG-GIRLRG stock powders were dissolved in ammonium acetate buffer $(0.1 \mathrm{M})$ to obtain a $5 \mathrm{mg} / \mathrm{mL}$ solution. ${ }^{111} \mathrm{InCl}_{3}\left(370 \mathrm{MBq} \cdot \mathrm{mL}^{-1}\right.$ in $\left.0.5 \mathrm{M} \mathrm{HCl}, \mathrm{pH} 1.1-1.4\right)$ was obtained from Mallinckrodt Pharmaceuticals. Ammonium acetate $(400 \mu \mathrm{L}$ of $0.5 \mathrm{M})$ was added to ${ }^{111} \mathrm{InCl}_{3}$ stock solution $(450 \mu \mathrm{L})$ and carefully mixed; the final $\mathrm{pH}$ was between 5.5 and 5.8. The ${ }^{111} \mathrm{InCl}_{3}$ was then added to the DTPA-PEG control and DTPA-PEG-GIRLRG at a ratio of $370: 1 \mathrm{kBq}: \mu \mathrm{g}$, and the reaction mixture was incubated at $95^{\circ} \mathrm{C}$ with constant shaking for $1 \mathrm{~h}$. The radiolabeling efficiency of the PEG peptides was determined using instant thin-layer chromatography, and labeled peptides of $95 \%$ purity or greater were used for in vivo studies. Dynamic light-scattering studies were performed with the PEGylated peptides to confirm the absence of aggregates or changes in size (data not shown).

In Vitro Binding Assay with GRP78. GRP78 protein $(20 \mu \mathrm{g})$ in a volume of $100 \mu \mathrm{L}$ of binding buffer (10 mM HEPES, $5 \mathrm{mM} \mathrm{MgCl}$, $1 \mathrm{mM}$ EDTA, $0.1 \%$ bovine serum albumin, leupeptin $[10 \mu \mathrm{g} / \mathrm{mL}]$, pepstatin $[10 \mu \mathrm{g} / \mathrm{mL}$ ], aprotinin $[0.5 \mu \mathrm{g} / \mathrm{mL}]$, and bacitracin $[200 \mu \mathrm{g} / \mathrm{mL}]$, $\mathrm{pH}$ 7.4) was applied to $0.1 \%$ polyethyleneimine-pretreated wells of a 96-well Multiscreen Durapore filtration plate (Millipore Corp.) via vacuum manifold aspiration. The wells were washed 3 times with wash buffer (10 mM HEPES, $5 \mathrm{mM} \mathrm{MgCl} 2,1 \mathrm{mM}$ EDTA, $0.1 \%$ bovine serum albumin, $\mathrm{pH}$ 7.4). To this, $10 \mu \mathrm{L}$ of binding buffer with and without $20 \mu \mathrm{g}$ of GRP78 peptide (nonradiative PEG-DTPA-GIRLRG) were added to block and nonblock wells, respectively. Approximately 20,000 counts per minute of ${ }^{111}$ In-DTPA-PEG-GIRLRG were added in a volume of $100 \mu \mathrm{L}$ to each well. The plate was incubated at room temperature for $1 \mathrm{~h}$ and then washed twice with wash buffer. The membranes were allowed to dry and placed in separate tubes for determination of bound radioactivity. Radioactivity was counted using an automated $\gamma$-counter (Packard II; Perkin Elmer).

\section{Serum Stability Studies}

In vitro serum stability of the ${ }^{111}$ In-radiolabeled complexes was performed to determine whether the compounds radiolabeled with ${ }^{111} \mathrm{In}$ 


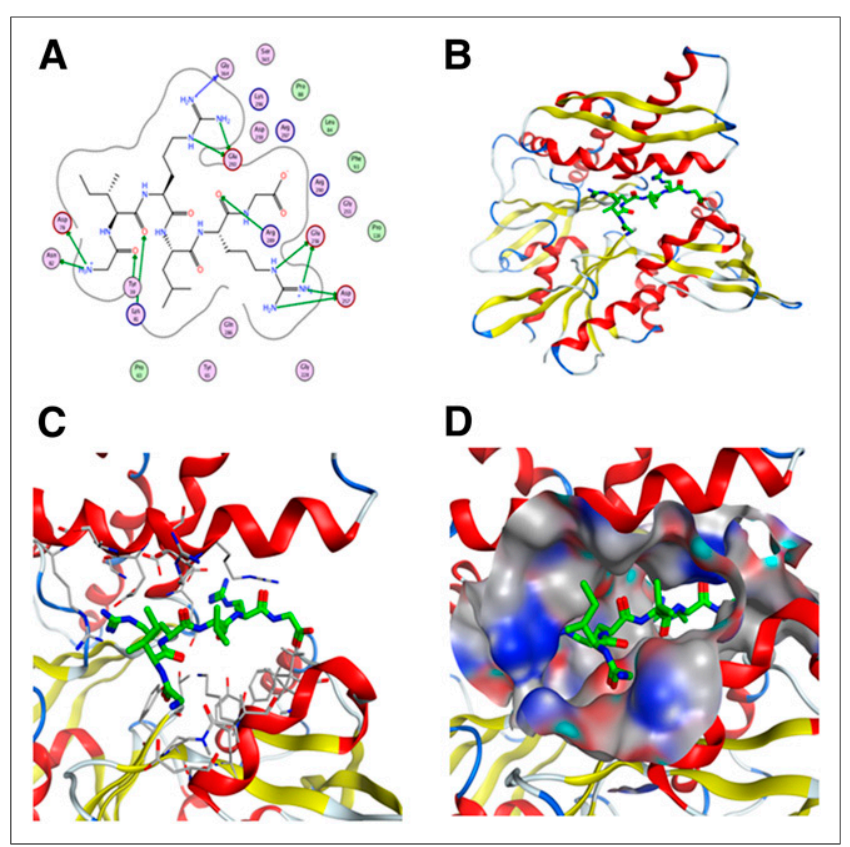

FIGURE 1. Modeling of GIRLRG peptide into GRP78 ATPase domain. (A) Interaction map between GIRLRG peptide and GRP78 residues (circles). Position of GIRLRG (green) in ATPase domain (B) with interacting residues (gray) $(C)$ and binding site surface (hydrophobic areas in gray, oxygen in red and nitrogen in blue) (D).

remained stable in the mouse serum. Ten microliters $(3.7 \mathrm{MBq}$ $[\sim 100 \mu \mathrm{Ci}])$ of ${ }^{111}$ In-labeled PEG control and PEG-GIRLRG were added to $90 \mu \mathrm{L}$ of mouse serum and incubated at $37^{\circ} \mathrm{C}$ with agitation (300 rpm). Aliquots were removed at each time point $(0.5,1,2,4,24$, 48 , and $72 \mathrm{~h}$ ) and analyzed using instant thin-layer chromatography with $50 \mathrm{mM}$ DTPA solution as the mobile phase. All reactions were conducted in triplicate and data plotted using GraphPad Prism software (GraphPad).

\section{Mice}

All animal studies were performed in accordance with the guidelines of the Institutional Animal Care and Use Committee and with protocols approved by the Washington University Division of Comparative Medicine. Tumor models were established in 6- to 8-wk-old female athymic nude mice (obtained from Harlan Laboratories, USA) by subcutaneous injection of tumor cells $\left(1-3 \times 10^{6}\right)$ into both hind limbs for HT3 and OE33 heterotopic tumor models and the right hind limb only in the A549, BxPC3, and D54 heterotopic tumor models. The tumors were grown until a size of $1 \mathrm{~cm}^{3}$ before being used for SPECT imaging. Before injection with radiolabeled peptide, the right hind limb was irradiated with $3 \mathrm{~Gy}, 3$ times over $24 \mathrm{~h}$, using the RadSource RS2000 Biologic Systems irradiator. The rest of the body (including the left hind limb tumor) was blocked from irradiation using lead plates.

\section{Nano-SPECT/CT Imaging and Biodistribution Studies}

Mice ( $n=3$ per group) were injected intravenously with approximately $18.5 \mathrm{MBq}(500 \mu \mathrm{Ci})$ of ${ }^{111}$ In-DTPA-PEG-GIRLRG or PEG control in a volume of $100 \mu \mathrm{L}$. Whole-body SPECT images were obtained at 48 and $72 \mathrm{~h}$ after injection using a NanoSPECT/CT imager (Bioscan Inc.) fitted with 2-mm pinhole collimators in helical scanning mode. Mice were placed prone and scanned under anesthesia $(0.5 \mathrm{~L} / \mathrm{min}$ $1.5 \%$ isoflurane in air). A $45-\mathrm{keV}$ helical CT scan was obtained first, and then SPECT was performed at 24 projections and $60 \mathrm{~s}$ per projection, for a total scan time of about $45 \mathrm{~min}$. Tomographic data were reconstructed iteratively with the manufacturer-supplied InVivoScope and HiSPECT software for CT and SPECT, respectively.

After scanning, $96 \mathrm{~h}$ after injection, the biodistribution of the labeled peptide in various organs was determined. The animals were sacrificed, and organs of interest including blood were dissected/collected, weighed, and counted in a $\gamma$-counter along with a standard of the injected activity to allow calculation of the injected dose per organ.

\section{Statistical Analysis}

Quantitative data are expressed as mean \pm SD. Means were compared using the Student $t$ test. $P$ values of less than 0.05 were considered statistically significant.

\section{RESULTS}

\section{GIRLRG Binds to ATPase Domain of GRP78 Protein}

We first wanted to identify the binding site of the GIRLRG peptide in the GRP78 protein. For this, we performed in silico modeling studies of the GIRLRG peptide, with GRP78 showing the peptide's binding sites and interacting residues within the ATPase domain of the GRP78 protein (Fig. 1). Figure 1A shows the interaction map between GIRLRG peptide and GRP78 residues. In this model, the 2 arginine (Arg) residues of GIRLRG fit on either side of the main central helix in GRP78 and are surrounded by charged residues (Figs. 1B and 1C). The first Arg of GIRLRG interacts strongly with Asp 259 and Glu 293, forming a charge network also with Lys 296 and Arg 297 (Figs. 1B and 1C). The second peptide Arg is flanked by Glu 256 and A257. The residues in GRP78 that interact with GIRLRG peptide are Thr 38, Tyr 39, Pro 63, Tyr 65, Asp 78, Lys 81, Asn 82, Leu 84, Phe 93, Glu 256, Asp257, Asp 259, Arg 289, Arg 290, Lys 294, Lys 296, and Arg 297. The GRP78 binding site surface is shown in Figure 1D.

\section{GIRLRG Peptide Binds Specifically to GRP78 Protein}

To determine the binding affinity of GIRLRG peptide to GRP78 protein, we used SPR technology. GRP78 protein was immobilized on the surface of the CM4 sensor chip, and approximately 7,000 resonance units of GRP78 protein were immobilized (Fig. 2A). The GIRLRG peptide was dissolved in HBS-EP buffer at various concentrations $(10,5,2.5,1.25$, and $0.313 \mathrm{mM})$ and passed over the immobilized GRP78 for $2 \mathrm{~min}$ (Fig. 2B). The rate constant was obtained by fitting the sensogram data after reference subtraction (data from blank channel) using the BIA evaluation 3.0 software. The change in resonance units with varying concentrations of peptide indicated the change in bound mass on sensor surface with time, and the dissociation constant was found to be $2.16 \times 10^{-3} \mathrm{M}$.

\section{GIRLRG Peptide Binds to Cancer Cells In Vitro}

Five cancer cell lines, D54 (glioma), HT3 (cervical), OE33 (esophageal), BxPC3 (pancreatic cancer), and A549 (lung cancer), were used to evaluate binding of GIRLRG peptide. Cancer cells were incubated with FITC-conjugated GIRLRG peptide or FITCconjugated scrambled peptide, and images were acquired using a fluorescent microscope. FITC-labeled GIRLRG peptide showed binding to D54, HT3, OE33, BxPC3, and A549 cell lines, and there was little or no binding of FITC-conjugated scrambled peptide (Fig. 3).

\section{Radiolabeling of PEG Peptides}

The radiolabeling of PEG peptides was optimized by varying the $\mathrm{pH}$, buffer, temperature, and amount of ${ }^{111} \mathrm{InCl}_{3}$ added per milligram of DTPA-PEG compounds. For all in vivo studies, 


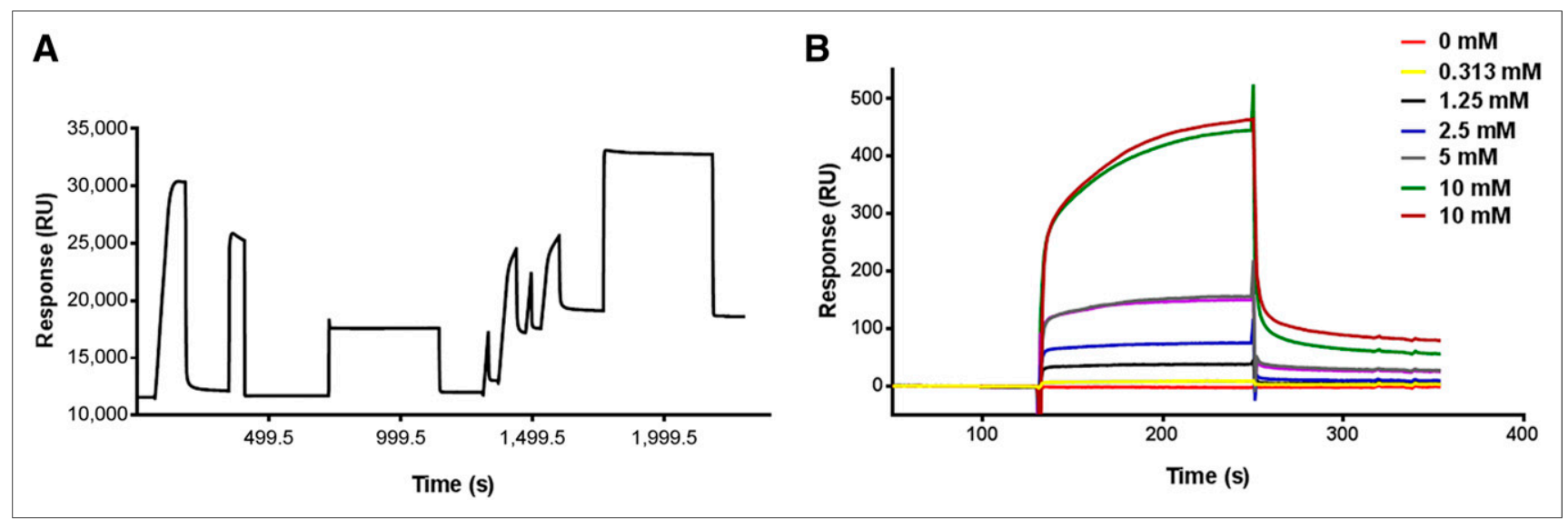

FIGURE 2. Binding affinity of GIRLRG peptide to GRP78 protein using SPR. (A) Sensogram for immobilization of GRP78 protein on surface of sensor chip via amine coupling method. (B) Overlay sensograms of indicated concentrations of GIRLRG peptide that passed over chip.

DTPA-PEG control and DTPA-PEG-GIRLRG peptide were labeled with ${ }^{111}$ In. The labeling efficiency was optimized by trying different temperatures, buffers, and $\mathrm{pH}$. The best yield was obtained by carrying out all reactions under absolute metal-free conditions. The optimal $\mathrm{pH}$ was 5.5, the optimal temperature was $95^{\circ} \mathrm{C}$, and ammonium acetate buffer resulted in the best labeling yield (Supplemental Table 1; supplemental materials are available at http://jnm.snmjournals.org). Dynamic light-scattering studies were performed with the PEGylated peptides to confirm the absence of aggregates or changes in size (data not shown). DTPA-PEG compounds were radiolabeled with a specific activity of $370 \mathrm{MBq}$ $(10 \mathrm{mCi})(>95 \%$ radiolabeling efficiency) per milligram of compound. The instant thin-layer chromatogram of ${ }^{111}$ In-DTPA compounds is shown in Supplemental Figure 1. To determine whether labeling $\left({ }^{111} \mathrm{In}\right)$ affected binding affinity for the target (GRP78), we performed an in vitro binding assay. We observed specific binding $(P<0.04)$ of the ${ }^{111}$ In-labeled GIRLG to GRP78 when the nonlabeled peptide (cold) alone was used as a competitor, indicating that labeling did not have any impact on binding affinity for the target (Supplemental Fig. 2).

\section{GIRLRG Peptide Binds Specifically to Tumors In Vivo}

In a pilot study, we compared the circulation of 5-, 10-, and 40$\mathrm{kDa}$ PEG and found 40-kDa PEG to have significantly longer blood circulation than 5- and 10-kDa PEG (data not shown). We therefore used 40-kDa PEG to enhance the blood circulation of the peptide constructs. To evaluate the efficacy of cancer-specific binding of ${ }^{111}$ In-labeled PEG-GIRLRG peptide, we noninvasively imaged cervical (HT3) and esophageal (OE33) heterotopic tumors using nano-SPECT/CT. Nude mice bearing HT3 and OE33 tumors in both the hind limbs were used $\left(\sim 1 \mathrm{~cm}^{3}\right)$. To evaluate the metabolic stability of the ${ }^{111}$ In labeled peptides, we performed a serum stability assay in vitro. ${ }^{111} \mathrm{In}$-labeled PEG-GIRLRG peptide was stable at all the time points $(0.5,1,2,4,24,48$, and $72 \mathrm{~h})$ tested (Supplemental Fig. 3). The PEG control and PEG-GIRLRG- ${ }^{111}$ In complexes were observed to be $99.2 \% \pm 0.6 \%$ and $97.7 \% \pm 0.4 \%$ intact in serum at $72 \mathrm{~h}$.

Because radiotherapy is the mainstay for treating local cancers including cervical and esophageal cancers, we also irradiated the tumors to be certain that selective binding in cancer was not disrupted during therapy. The tumor on the right hind limb was irradiated with 3 fractions of 3 Gy over a course of $24 \mathrm{~h}$; the tumor on the left hind limb was used as sham control. The first 2 fractions of $3 \mathrm{~Gy}$ were $6 \mathrm{~h}$ apart, and the third fraction was $12 \mathrm{~h}$ apart. The mice were then injected with $18.5 \mathrm{MBq}(0.37 \mathrm{MBq} / \mu \mathrm{g}[500 \mu \mathrm{Ci}$; $10 \mu \mathrm{Ci} / \mu \mathrm{g}])$ of radiolabeled peptide via the tail vein. In pilot experiments, we performed SPECT imaging of the mice at 24, 48, 72, and $96 \mathrm{~h}$. We found optimal binding of PEG-GIRLRG to tumors at 48 and $72 \mathrm{~h}$ after injection. ${ }^{111}$ In-labeled PEG-DTPA-GIRLRG peptide bound to both HT3 (Fig. 4A) and OE33 (Fig. 5A). Very low or negligible binding of ${ }^{111}$ In-labeled PEG-DTPA control was observed in both HT3 (Fig. 4A) and OE33 (Fig. 5A) tumors. Irradiating the tumors (right hind limb) did not affect tumor binding of the radiolabeled GIRLRG peptide to either HT3 or OE33 tumors.

Post-SPECT biodistribution was done $96 \mathrm{~h}$ after injection (Figs. 4 and 5). The post-SPECT biodistribution data of the ${ }^{111} \mathrm{In}$ labeled compounds in tumor-bearing mice are summarized in Figures 4B and $5 \mathrm{~B}$. The biodistribution results correlate with the SPECT imaging data. Significantly higher $(P<0.001)$ uptake of the radiolabeled GIRLRG was observed in tumors than with control peptide. The radiolabeled peptides were observed in circulation as seen from the blood uptake in the biodistribution data. Labeled peptide was also observed in the liver of both HT3 and OE33 tumor-bearing mice as these peptides were being cleared from the circulation. The levels of the labeled peptide in other organs were not significant.

In addition, we also screened lung cancer (A549), pancreatic cancer (BxPC3), and glioblastoma (D54) tumor models for binding of radiolabeled GIRLRG peptide. SPECT/CT imaging at $48 \mathrm{~h}$ showed enhanced binding of ${ }^{111}$ In-labeled PEG-DTPA-GIRLRG in A549, BxPC3, and D54 tumors (Fig. 6).

\section{DISCUSSION}

Peptide receptors are overexpressed in cancers and have been used as molecular targets $(2,24)$. Imaging probes currently being developed for these receptors include somatostatin analogs, cholecystokinin/gastrin and GLP-1 analogs for neuroendocrine tumors, bombesin and neuropeptide-Y analogs for prostate or breast cancers, and Arg-Gly-Asp peptides for neoangiogenesis labeling $(2,24)$. The first and most successful Food and Drug Administrationapproved peptide-based radiopharmaceutical is the somatostatin analog (25) ${ }^{111}$ In-DTPA-octreotide $\left({ }^{111} \mathrm{In}\right.$-OctreoScan, ${ }^{111} \mathrm{In}$-pentetreotide) $(2,26,27)$. It is being used for imaging somatostatin receptor-positive lesions, such as neuroendocrine tumors, 


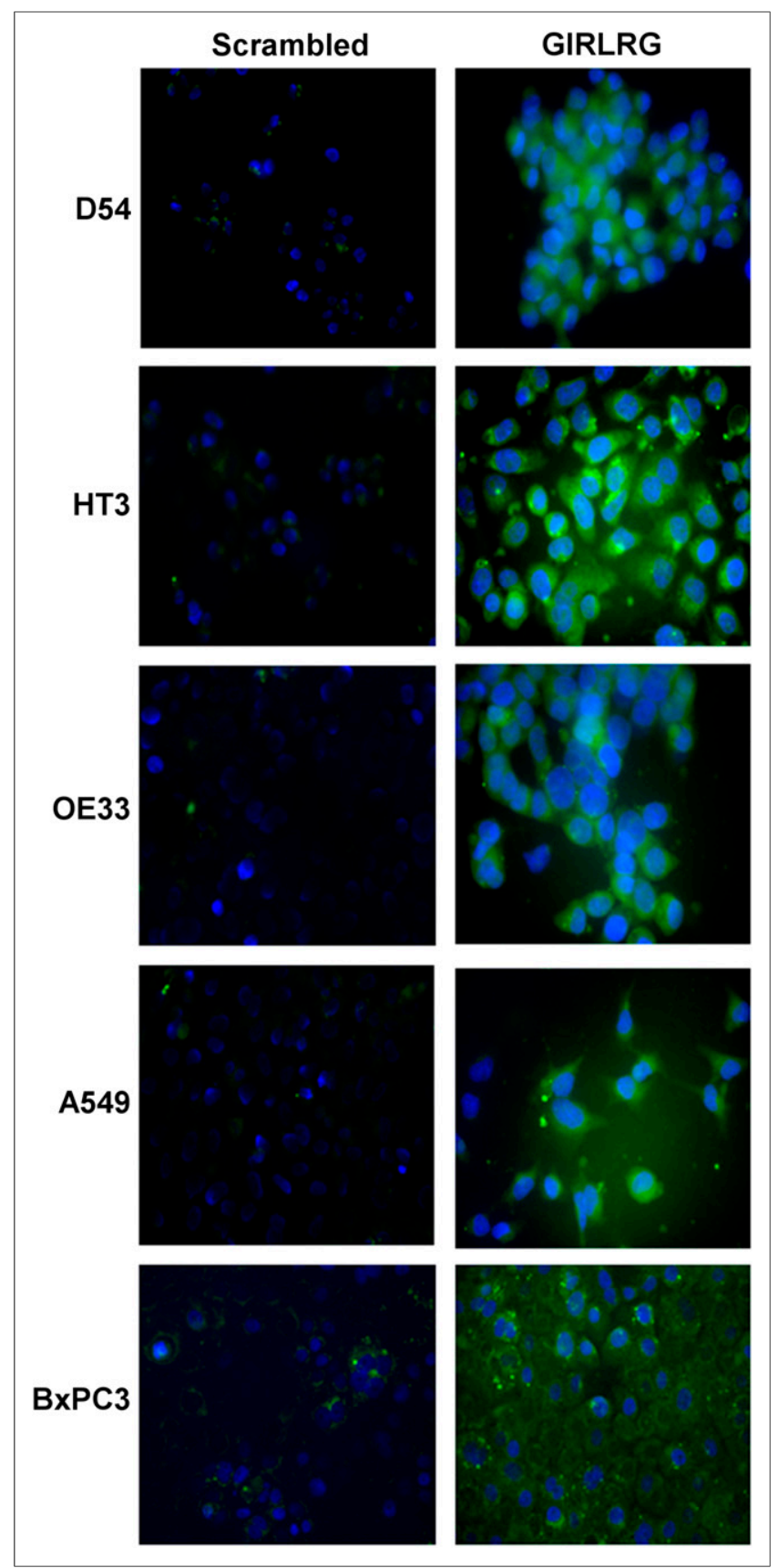

FIGURE 3. Binding of FITC-conjugated scrambled and FITC-conjugated GIRLRG peptide to various cancer cell lines as observed under fluorescent microscope (resolution, 200x).

mammary cancer, and small cell lung cancer (25-27). The successful clinical application of this radiopharmaceutical raised interest in the development of radiolabeled peptides to target other tumorrelated peptide receptor systems.

Increasing evidence suggests that glucose-related stress response proteins serve as functional chaperones on the surface of cancer cells. These regulate multiple signaling pathways related to apoptosis, immune function, and drug resistance. GRP78 is one such surface protein that could be potentially developed for targeted therapy against various cancers (5). GRP78 plays a vital role in the unfolded protein response, which regulates survival or death pathways in response to ER stress. GRP78 reestablishes normal function of the cell by translation repression, reduction of intermediate protein aggregates, removal of improperly folded proteins, and regulation of intracellular $\mathrm{Ca}^{2+}(28)$. GRP78 is reported to be expressed on the cell surface of tumors (4). GIRLRG peptide was identified by in vivo biopanning (3). We have shown that GIRLRG bound specifically to GRP78 on the surface of the cancer cells both in vitro and in vivo. Further, when these cells were treated with antibodies specific to GRP78 (blocking antibody), we observed decreased binding of GIRLRG to these tumors both in vitro and in vivo. The binding of GIRLRG peptide was related to the expression of GRP78 in vitro and in vivo (3). GRP78-targeting peptides conjugated with cytotoxic agents have been shown to specifically bind tumors and induce cytotoxicity $(9,15)$. GIRLRG peptide was conjugated to paclitaxel (sustainedrelease nanoparticle) that specifically targeted breast cancer and glioblastoma and delayed tumor growth (3). In the present study, we sought to identify the binding domain of GIRLRG peptide to GRP78 protein. Using molecular modeling and docking studies, we found that the GIRLRG peptide bound to the residues in the ATPase domain of GRP78. Different peptides and antibodies binding to the ATPase and substrate binding domains of GRP78 have been reported $(5,29-32)$. Some of these have been shown to positively or negatively regulate the growth-promoting effects of GRP78, although their modes of action have not been extensively investigated. We used SPR to evaluate the affinity of GIRLRG to GRP78. SPR analysis showed that GIRLRG had a rate constant of $2.16 \times 10^{-3} \mathrm{M}$ for GRP78. One of the reasons for low binding affinity with SPR could be that GIRLRG peptide binding to the ATPase domain might require additional cofactors, which were

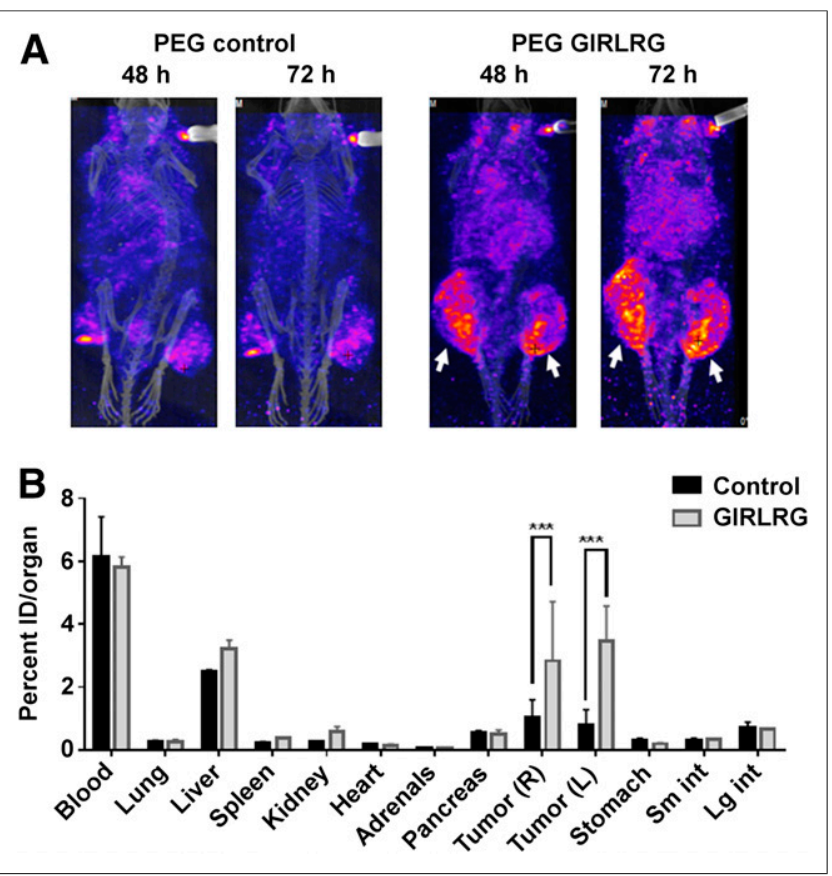

FIGURE 4. SPECT imaging (A) and post-SPECT biodistribution (B) with radiolabeled PEG-GIRLRG and PEG control peptide in nude mice with heterotopic cervical tumors (HT3). Tumor on right hind limb was irradiated with 3 doses of $3 \mathrm{~Gy}$, and left was sham control. Enhanced tumor binding of PEG-GIRLRG peptide is observed in HT3 tumors at both 48 and $72 \mathrm{~h}$ after injection (white arrows). ${ }^{\star \star \star} P<0.001$. 
not available in this assay but might be available in vivo. Indeed, we did show FITC-conjugated GIRLRG peptide bound to glioma (D54), cervical (HT3), esophageal (OE33), and lung (A549) cancer cells in vitro.

We evaluated the GIRLRG peptide as an imaging probe for various tumors. Peptides are small and could be cleared quickly from the system, leading to poor bioavailability in targeted tissues (33). To overcome this problem, we conjugated the GIRLRG peptide to 40-kDa PEG to enhance its circulation time and extravasation to the tumors, taking advantage of enhanced permeability and retention. This strategy of PEGylation has been successfully used and approved for various biomolecules used in the clinic (34). PEG-GIRLRG was radiolabeled with ${ }^{111}$ In using DTPA as the chelator. The background signal was evaluated using a 40-kDa PEG construct having the chelator DTPA (PEG control). PEG-GIRLRG or PEG control was used for imaging of cervical (HT3) and esophageal (OE33) heterotopic tumors with nano-SPECT at 24, 48, 72, and $96 \mathrm{~h}$. At 24-h SPECT imaging, we found the animals showed increased background because the PEG-GIRLRG was still circulating throughout the body. We found good tumor-selective binding efficacy at 48 and $72 \mathrm{~h}$ because these times showed optimal binding of the radiolabeled peptide. The imaging at $96 \mathrm{~h}$ was diminished as compared with 48 and $96 \mathrm{~h}$ because of reduced activity. PEG-GIRLRG specifically bound to HT3 and OE33 tumors whereas little or no binding was observed in PEG control. These data indicated that PEG-GIRLRG had specific binding to the HT3 and OE33 tumors, most likely by binding to the GRP78 on the surface of the tumors. Because radiation is routinely used in treating cervical and esophageal cancers, we compared irradiated tumors to

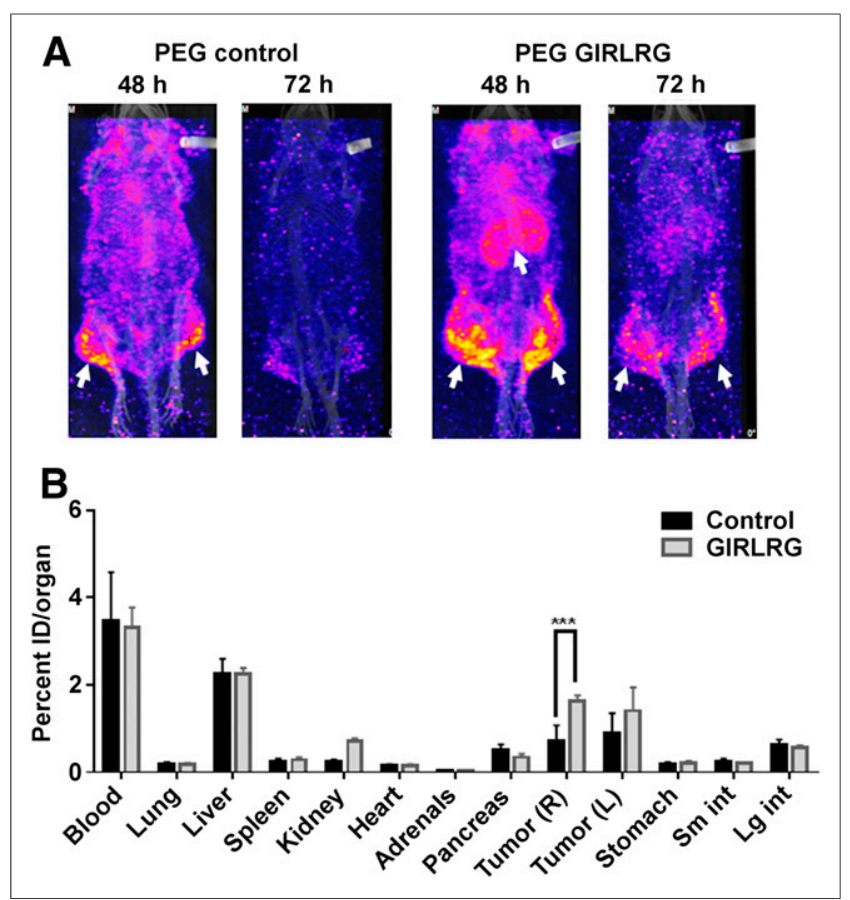

FIGURE 5. SPECT imaging (A) and post-SPECT biodistribution (B) with radiolabeled PEG-GIRLRG and PEG control peptide in nude mice with heterotopic esophageal (OE33). Tumor on right hind limb was irradiated with 3 doses of $3 \mathrm{~Gy}$, and left was sham control. Enhanced tumor binding of PEG-GIRLRG peptide is observed in HT3 tumors at both 48 and $72 \mathrm{~h}$ after injection (white arrows). ${ }^{\star \star \star} P<0.001$.

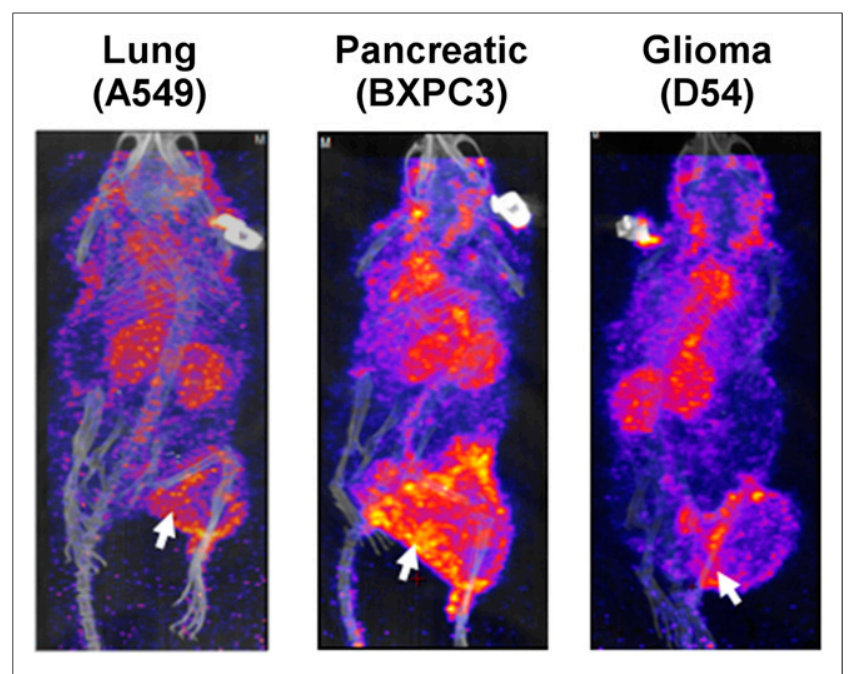

FIGURE 6. SPECT imaging ( $48 \mathrm{~h}$ after injection) with radiolabeled PEG-GIRLRG peptide in nude mice with heterotopic lung (A549), pancreatic (BXPC3), and brain (D54) cancer. Tumor on right hind limb was irradiated with 3 doses of 3 Gy. Enhanced tumor binding of PEGGIRLRG peptide is observed in A549, BXPC3, and D54 tumors (white arrows).

sham irradiated tumors. Irradiation of tumors did not affect the specific binding of PEG-GIRLRG peptide to the HT3 (cervical) and OE33 (esophageal) tumors. Irradiation induces surface expression of GRP78 on tumor cells. Enhanced binding of the PEG-GIRLRG peptide was not observed in the irradiated tumor compared with the sham irradiated tumor. This could be attributed to an abscopal effect, which may have led to similar binding of the PEG-GIRLRG peptide to the sham and irradiated tumor $(35,36)$.

Post-SPECT biodistribution data further supported the SPECT imaging data. Significantly higher uptake of PEG-GIRLRG peptide was observed in tumors than the PEG control. At $96 \mathrm{~h}$, both the PEG-GIRLRG and the PEG-control peptides were still observed in the blood and liver, implying that this is a function of the PEG moiety and not the peptide itself. The higher blood and liver levels may be reduced by optimizing the size of the PEG moiety. In addition to the cervical and esophageal cancer, we evaluated binding of PEG-GIRLRG in lung cancer (A549), pancreatic cancer (BXPC3), and glioblastoma (D54) models. Similar to the cervical and esophageal tumors, we found specific binding of PEG-GIRLRG to lung cancer, pancreatic cancer, and glioblastoma.

\section{CONCLUSION}

Overall, this study offers further support for surface GRP78 as a peptide receptor and a molecular target for development of diagnostics and therapeutics for various cancers. Our long-term aim is to develop GIRLRG peptide as a carrier for therapeutic agents. In this study, we showed specifically that GIRLRG binds to GRP78 on the surface of cancer cells and that this could be a potential peptide for targeting various cancers such as cervical, esophageal, lung, glioma, and pancreatic. Presently PEG-GIRLRG is being further optimized to improve the long-term biodistribution and being prepared for preclinical efficacy and safety testing before moving it to clinical trials. 


\section{DISCLOSURE}

The costs of publication of this article were defrayed in part by the payment of page charges. Therefore, and solely to indicate this fact, this article is hereby marked "advertisement" in accordance with 18 USC section 1734. This work was supported by National Cancer Institute grants 1R01CA140220-02, 5R01CA125757-06, 7R01CA112385-0 (Dennis E. Hallahan), Siteman Cancer Research Award, Elizabeth and James McDonnell III Endowment (Dennis E. Hallahan), American Association for Cancer Research grant 12-60-26-HALL (Dennis E. Hallahan), The Barnes-Jewish Hospital Foundation (BJHF), and the Washington University Institute of Clinical and Translational Sciences (ICTS) grant (Dennis E. Hallahan) and Department of Radiation Oncology Startup Funds (Dinesh Thotala). No other potential conflict of interest relevant to this article was reported.

\section{ACKNOWLEDGMENTS}

We acknowledge the Siteman Cancer Center Small Animal Imaging Core and we thank Nicole Fettig, Amanda Klaas, and Cedric Mpoy for their technical assistance.

\section{REFERENCES}

1. de Barros AB, Tsourkas A, Saboury B, Cardoso VN, Alavi A. Emerging role of radiolabeled nanoparticles as an effective diagnostic technique. EJNMMI Res. 2012;2:39.

2. Schottelius M, Wester HJ. Molecular imaging targeting peptide receptors. Methods. 2009;48:161-177.

3. Passarella RJ, Spratt DE, van der Ende AE, et al. Targeted nanoparticles that deliver a sustained, specific release of Paclitaxel to irradiated tumors. Cancer Res. 2010;70:4550-4559.

4. Lee AS. GRP78 induction in cancer: therapeutic and prognostic implications. Cancer Res. 2007;67:3496-3499.

5. Lee AS. Glucose-regulated proteins in cancer: molecular mechanisms and therapeutic potential. Nat Rev Cancer. 2014;14:263-276.

6. Zhang Y, Liu R, Ni M, Gill P, Lee AS. Cell surface relocalization of the endoplasmic reticulum chaperone and unfolded protein response regulator GRP78/ BiP. J Biol Chem. 2010;285:15065-15075.

7. Delpino A, Castelli M. The $78 \mathrm{kDa}$ glucose-regulated protein (GRP78/BIP) is expressed on the cell membrane, is released into cell culture medium and is also present in human peripheral circulation. Biosci Rep. 2002;22:407-420.

8. Triantafilou M, Fradelizi D, Triantafilou K. Major histocompatibility class one molecule associates with glucose regulated protein (GRP) 78 on the cell surface. Hum Immunol. 2001;62:764-770.

9. Arap MA, Lahdenranta J, Mintz PJ, et al. Cell surface expression of the stress response chaperone GRP78 enables tumor targeting by circulating ligands. Cancer Cell. 2004;6:275-284.

10. Fu Y, Lee AS. Glucose regulated proteins in cancer progression, drug resistance and immunotherapy. Cancer Biol Ther. 2006;5:741-744.

11. Mintz PJ, Kim J, Do KA, et al. Fingerprinting the circulating repertoire of antibodies from cancer patients. Nat Biotechnol. 2003;21:57-63.

12. Kern J, Untergasser G, Zenzmaier C, et al. GRP-78 secreted by tumor cells blocks the antiangiogenic activity of bortezomib. Blood. 2009;114:39603967.

13. Ma X, Guo W, Yang S, Zhu X, Xiang J, Li H. Serum GRP78 as a tumor marker and its prognostic significance in non-small cell lung cancers: a retrospective study. Dis Markers. 2015;2015:814670.
14. Rasche L, Duell J, Morgner C, et al. The natural human IgM antibody PAT-SM6 induces apoptosis in primary human multiple myeloma cells by targeting heat shock protein GRP78. PLoS One. 2013;8:e63414.

15. Liu Y, Steiniger SC, Kim Y, Kaufmann GF, Felding-Habermann B, Janda KD. Mechanistic studies of a peptidic GRP78 ligand for cancer cell-specific drug delivery. Mol Pharm. 2007;4:435-447.

16. Lieber M, Smith B, Szakal A, Nelson-Rees W, Todaro G. A continuous tumorcell line from a human lung carcinoma with properties of type II alveolar epithelial cells. Int J Cancer. 1976;17:62-70.

17. Bigner SH, Bullard DE, Pegram CN, Wikstrand CJ, Bigner DD. Relationship of in vitro morphologic and growth characteristics of established human gliomaderived cell lines to their tumorigenicity in athymic nude mice. $J$ Neuropathol Exp Neurol. 1981;40:390-409.

18. Rockett JC, Larkin K, Darnton SJ, Morris AG, Matthews HR. Five newly established oesophageal carcinoma cell lines: phenotypic and immunological characterization. Br J Cancer. 1997;75:258-263.

19. Tan MH, Nowak NJ, Loor R, et al. Characterization of a new primary human pancreatic tumor line. Cancer Invest. 1986;4:15-23.

20. Fogh J, Wright WC, Loveless JD. Absence of HeLa cell contamination in 169 cell lines derived from human tumors. J Natl Cancer Inst. 1977;58:209-214.

21. Wisniewska M, Karlberg T, Lehtio L, et al. Crystal structures of the ATPase domains of four human Hsp70 isoforms: HSPA1L/Hsp70-hom, HSPA2/Hsp70-2, HSPA6/Hsp70B', and HSPA5/BiP/GRP78. PLoS One. 2010;5:e8625.

22. Wang JM, Cieplak P, Kollman PA. How well does a restrained electrostatic potential (RESP) model perform in calculating conformational energies of organic and biological molecules? J Comput Chem. 2000;21:1049-1074.

23. Kapoor V, Singh AK, Dey S, Sharma SC, Das SN. Circulating cycloxygenase-2 in patients with tobacco-related intraoral squamous cell carcinoma and evaluation of its peptide inhibitors as potential antitumor agent. J Cancer Res Clin Oncol. 2010;136:1795-1804.

24. Fani M, Maecke HR, Okarvi SM. Radiolabeled peptides: valuable tools for the detection and treatment of cancer. Theranostics. 2012;2:481-501.

25. Ambrosini V, Fani M, Fanti S, Forrer F, Maecke HR. Radiopeptide imaging and therapy in Europe. J Nucl Med. 2011;52(suppl 2):42S-55S.

26. Rufini V, Calcagni ML, Baum RP. Imaging of neuroendocrine tumors. Semin Nucl Med. 2006;36:228-247.

27. Kaltsas GA, Papadogias D, Makras P, Grossman AB. Treatment of advanced neuroendocrine tumours with radiolabelled somatostatin analogues. Endocr Relat Cancer. 2005;12:683-699.

28. Ma Y, Hendershot LM. The role of the unfolded protein response in tumour development: friend or foe? Nat Rev Cancer. 2004;4:966-977.

29. Gonzalez-Gronow M, Kaczowka SJ, Payne S, Wang F, Gawdi G, Pizzo SV. Plasminogen structural domains exhibit different functions when associated with cell surface GRP78 or the voltage-dependent anion channel. J Biol Chem. 2007;282:32811-32820.

30. Raiter A, Weiss C, Bechor Z, et al. Activation of GRP78 on endothelial cell membranes by an ADAM15-derived peptide induces angiogenesis. $J$ Vasc Res. 2010;47:399-411.

31. Gonzalez-Gronow M, Cuchacovich M, Llanos C, Urzua C, Gawdi G, Pizzo SV. Prostate cancer cell proliferation in vitro is modulated by antibodies against glucose-regulated protein 78 isolated from patient serum. Cancer Res. 2006;66:11424-11431.

32. Maddalo D, Neeb A, Jehle K, et al. A peptidic unconjugated GRP78/BiP ligand modulates the unfolded protein response and induces prostate cancer cell death. PLoS One. 2012;7:e45690.

33. Yamamoto Y, Tsutsumi Y, Mayumi T. Molecular design of bioconjugated cell adhesion peptide with a water-soluble polymeric modifier for enhancement of antimetastatic effect. Curr Drug Targets. 2002;3:123-130.

34. Bailon P, Won CY. PEG-modified biopharmaceuticals. Expert Opin Drug Deliv. 2009;6:1-16.

35. Formenti SC, Demaria S. Systemic effects of local radiotherapy. Lancet Oncol. 2009; 10:718-726.

36. Siva S, MacManus MP, Martin RF, Martin OA. Abscopal effects of radiation therapy: a clinical review for the radiobiologist. Cancer Lett. 2015;356:82-90. 